Volume 25 (2019) 55-63

DOI: $10.24330 /$ ieja. 504110

\title{
ON THE ASSOCIATED PRIMES OF THE $d$-LOCAL COHOMOLOGY MODULES
}

\author{
Z. Rahimi-Molaei, Sh. Payrovi and S. Babaei \\ Received: 23 January 2018; Revised: 29 July 2018; Accepted: 18 August 2018 \\ Communicated by A. Çiğdem Özcan

\begin{abstract}
This paper is concerned to relationship between the sets of associated primes of the $d$-local cohomology modules and the ordinary local cohomology modules. Let $R$ be a commutative Noetherian local ring, $M$ an $R$-module and $d, t$ two integers. We prove that $\operatorname{Ass}\left(H_{d}^{t}(M)\right)=\bigcup_{I \in \Phi} \operatorname{Ass}\left(H_{I}^{t}(M)\right)$ whenever $H_{d}^{i}(M)=0$ for all $i<t$ and $\Phi=\{I: I$ is an ideal of $\mathrm{R}$ with $\operatorname{dim} R / I \leq$ $d\}$. We give some information about the non-vanishing of the $d$-local cohomology modules. To be more precise, we prove that $H_{d}^{i}(R) \neq 0$ if and only if $i=n-d$ whenever $R$ is a Gorenstein ring of dimension $n$. This result leads to an example which shows that $\operatorname{Ass}\left(H_{d}^{n-d}(R)\right)$ is not necessarily a finite set.
\end{abstract}

Mathematics Subject Classification (2010): 13D45, 14B15

Keywords: Associated primes, vanishing theorem, $d$-local cohomology module

\section{Introduction}

Throughout this paper, $R$ denotes a commutative Noetherian ring with non-zero identity. For an ideal $I$ of $R$ and an $R$-module $M$, the $i$ th local cohomology module of $M$ with respect to $I$ is defined as

$$
H_{I}^{i}(M) \cong \underset{n \in \mathbb{N}}{\lim _{n \in}} \operatorname{Ext}_{R}^{i}\left(R / I^{n}, M\right) .
$$

The reader can refer to [6] for the basic properties of local cohomology modules. An important problem in commutative algebra is determining when the set of associated primes of the $i$ th local cohomology module $H_{I}^{i}(M)$ is finite. In [10], Huneke raised the following question: If $M$ is a finitely generated $R$-module, then the set of associated primes of $H_{I}^{i}(M)$ is finite for all ideals $I$ of $R$ and all $i \geq 0$. This problem has been studied by many authors, it was shown that it is true in many situations, for examples see $[4,5,8]$. In particular, it is shown in [5] that if for a finitely generated $R$-module $M$ and integer $t$, the local cohomology modules $H_{I}^{i}(M)$ are finitely generated for all $i<t$, then the set $\operatorname{Ass}\left(H_{I}^{t}(M)\right)$ is finite. There are several papers devoted to the extension of the above results to more 
general situations, for examples see $[1,9]$. However there are counterexamples which show that it is not true in general, for examples see $[11,16]$. The purpose of this paper is to make a counterexample to above question in the context of general local cohomology modules. The theory of general local cohomology modules over commutative Noetherian rings introduced by Bijan-Zadeh in [3]. General local cohomology theory described as follows.

Let $\Phi$ be a non-empty set of ideals of $R$. We call $\Phi$ a system of ideals of $R$ if, whenever $I, I^{\prime} \in \Phi$, then there exists $J \in \Phi$ such that $J \subseteq I I^{\prime}$. Such a system of ideals gives rise to an additive, left exact functor

$$
\Gamma_{\Phi}(M)=\{x \in M: I x=0 \text { for some ideal } I \in \Phi\}
$$

from the category of $R$-modules and $R$-homomorphisms to itself. $\Gamma_{\Phi}(-)$ is called the $\Phi$-torsion functor. For each $i \geq 0$, the $i$ th right derived functor of $\Gamma_{\Phi}(-)$ is denoted by $H_{\Phi}^{i}(-)$. For an ideal $I$ of $R$, if $\Phi=\left\{I^{n}: n \in \mathbb{N}\right\}$, then $H_{\Phi}^{i}(-)$ coincides with the ordinary local cohomology functor $H_{I}^{i}(-)$.

Let $d \geq 0$ be an integer. We denote $\Gamma_{\Phi}(-)$ and $H_{\Phi}^{i}(-)$ by $\Gamma_{d}(-)$ and $H_{d}^{i}(-)$ respectively, for the system of ideals $\Phi=\{I: I$ is an ideal of $\mathrm{R}$ with $\operatorname{dim} R / I \leq d\}$. The functor $\Gamma_{d}(-)$ was originally defined in [2] and the modules $H_{d}^{i}(M)$ were called $d$-local cohomology modules associated to $M$ were studied in [18,19]. After some preliminary results in Section 2, for an $R$-module $M$ and an integer $t$ we prove that

$$
\operatorname{Ass}\left(H_{d}^{t}(M)\right)=\bigcup_{I \in \Phi} \operatorname{Ass}\left(\operatorname{Ext}_{R}^{t}(R / I, M)\right)=\bigcup_{I \in \Phi} \operatorname{Ass}\left(H_{I}^{t}(M)\right)
$$

where $\Phi=\{I: I$ is an ideal of $\mathrm{R}$ with $\operatorname{dim} R / I \leq d\}$ and $H_{d}^{i}(M)=0$ for all $i<t$. In Section 3, we shall provide some results concerning the vanishing and non-vanishing of $d$-local cohomology modules: we shall prove that, over a local ring $R$, if the non-zero finitely generated $R$-module $M$ has (Krull) dimension $n$, then there exists an integer $i$ with $0 \leq i \leq d$ such that $H_{d}^{n-i}(M) \neq 0$. We shall also prove that, when $R$ is local Gorenstein of dimension $n$, then $H_{d}^{i}(R) \neq 0$ if and only if $i=n-d$. Furthermore, we shall prove that $H_{d}^{n-d}(R)$ is a non-Artinian flat module for which $\operatorname{Ass}\left(H_{d}^{n-d}(R)\right)=\{\mathfrak{p} \in \operatorname{Spec}(R): \operatorname{dim} R / \mathfrak{p}=d\}$. This result leads to an example which shows that the Huneke question is not true in the context of $d$-local cohomology modules.

\section{The associated primes}

It is our intention in this section to present the relationship between the sets of associated primes of the $d$-local cohomology modules and the ordinary local 
cohomology modules. So throughout this section, $R$ will denote a ring and $I$ is an ideal of $R, d$ is an integer and $\Phi=\{I: I$ is an ideal of $\mathrm{R}$ with $\operatorname{dim} R / I \leq d\}$.

Lemma 2.1. Let $M$ be an $R$-module and $t$ be an integer such that $H_{d}^{i}(M)=0$ for all $i<t$. Then the following statements are true:

(i) $H_{I}^{i}(M) \subseteq H_{d}^{i}(M)$ for all $I \in \Phi$ and $i \leq t$.

(ii) $H_{c}^{i}(M) \subseteq H_{d}^{i}(M)$ for all $c \leq d$ and $i \leq t$.

Proof. (i) Since $\operatorname{Hom}_{R}\left(R / I, \Gamma_{d}(M)\right) \cong \operatorname{Hom}_{R}(R / I, M)$ for all $I \in \Phi$ so, by [14, Theorem 11.38], the Grothendieck spectral sequence $E_{2}^{p, q}:=\operatorname{Ext}_{R}^{p}\left(R / I, H_{d}^{q}(M)\right)$ converges to $E^{p+q}:=\operatorname{Ext}_{R}^{p+q}(R / I, M)$. It follows that there is a finite filtration

$$
0=F^{q+1} E^{q} \subseteq F^{q} E^{q} \subseteq \cdots \subseteq F^{1} E^{q} \subseteq F^{0} E^{q}=E^{q}
$$

of $E^{q}$ such that $E_{\infty}^{p, q-p} \cong F^{p} E^{q} / F^{p+1} E^{q}$, for all $p=0,1, \cdots, q$. Because $E_{\infty}^{p, q-p}$ is a subquotient of $E_{2}^{p, q-p}$ so $E_{\infty}^{p, q}=0$, for all $q<t$. Thus $E_{\infty}^{0, q} \cong E^{q}$, for all $q \leq t$. On the other hand, for all $q \leq t$, by the sequence $0 \longrightarrow E_{2}^{0, q} \longrightarrow E_{2}^{2, q-1}$ and $E_{2}^{2, q-1}=0$ we have $E_{\infty}^{0, q} \cong E_{2}^{0, q}$. Thus $E^{q} \cong E_{2}^{0, q}$. Therefore, $\operatorname{Ext}_{R}^{i}(R / I, M) \cong$ $\operatorname{Hom}_{R}\left(R / I, H_{d}^{i}(M)\right)$ and so

$$
\Gamma_{I}\left(H_{d}^{i}(M)\right) \cong \lim _{n \in \mathbb{N}} \operatorname{Hom}_{R}\left(R / I^{n}, H_{d}^{i}(M)\right) \cong \lim _{n \in \mathbb{N}} \operatorname{Ext}_{R}^{i}\left(R / I^{n}, M\right) \cong H_{I}^{i}(M),
$$

for all $i \leq t$. The proof is therefore complete.

(ii) It is similar to that of (i).

Corollary 2.2. Let $M$ be an $R$-module and $t$ be an integer such that $H_{d}^{i}(M)=0$, for all $i<t$. Then the following statements are true:

(i) $\operatorname{Ass}\left(H_{I}^{i}(M)\right)=\operatorname{Ass}\left(H_{d}^{i}(M)\right) \cap V(I)$, for all $I \in \Phi$ and $i \leq t$.

(ii) $\operatorname{Ass}\left(H_{J}^{i}(M)\right) \subseteq \operatorname{Ass}\left(H_{I}^{i}(M)\right)$ for all $I, J \in \Phi$ with $I \subseteq J$ and $i \leq t$.

Proof. (i) By a similar argument to that of Lemma 2.1 (i), one can shows that $\operatorname{Hom}_{R}\left(R / I, H_{d}^{i}(M)\right) \cong \operatorname{Hom}_{R}\left(R / I, H_{I}^{i}(M)\right)$, for all $I \in \Phi$ and all $i \leq t$. Therefore, $\operatorname{Ass}\left(\operatorname{Hom}_{R}\left(R / I, H_{d}^{t}(M)\right)\right)=\operatorname{Ass}\left(\operatorname{Hom}_{R}\left(R / I, H_{I}^{t}(M)\right)\right)$. Thus $\operatorname{Ass}\left(H_{I}^{t}(M)\right)=$ $V(I) \cap \operatorname{Ass}\left(H_{d}^{t}(M)\right)$.

(ii) It is obvious by (i).

We say that an $R$-module $M$ is $d$-torsion if $\Gamma_{d}(M)=M$, and it is $d$-torsion free if $\Gamma_{d}(M)=0$. Note, if $M$ is $d$-torsion, then $\operatorname{Supp}(M) \subseteq \Phi$. Also, if $M$ is finitely generated of $\operatorname{dim} M=n$, then $M$ is $d$-torsion if and only if $n \leq d$. We are now in a position to prove that the main result of this section. 
Theorem 2.3. Let $M$ be an $R$-module and $t$ be an integer such that $H_{d}^{i}(M)=0$, for all $i<t$. Then

$$
\operatorname{Ass}\left(H_{d}^{t}(M)\right)=\bigcup_{\mathfrak{p} \in V(\Phi)} \operatorname{Ass}\left(\operatorname{Ext}_{R}^{t}(R / \mathfrak{p}, M)\right)=\bigcup_{\mathfrak{p} \in V(\Phi)} \operatorname{Ass}\left(H_{\mathfrak{p}}^{t}(M)\right),
$$

where $V(\Phi)=\operatorname{Spec}(R) \cap \Phi$.

Proof. First of all, we show that

$$
\operatorname{Ass}\left(H_{d}^{t}(M)\right)=\bigcup_{\mathfrak{p} \in V(\Phi)} \operatorname{Ass}\left(\operatorname{Hom}_{R}\left(R / \mathfrak{p}, H_{d}^{t}(M)\right)\right) .
$$

For an ideal $I$ of $R$, it is clear that $0:_{H_{d}^{t}(M)} I \cong \operatorname{Hom}_{R}\left(R / I, H_{d}^{t}(M)\right)$ thus $\operatorname{Ass}\left(H_{d}^{t}(M)\right) \supseteq \bigcup_{\mathfrak{p} \in V(\Phi)} \operatorname{Ass}\left(\operatorname{Hom}_{R}\left(R / \mathfrak{p}, H_{d}^{t}(M)\right)\right)$. Let $\mathfrak{p} \in \operatorname{Ass}\left(H_{d}^{t}(M)\right)$. Then there exists a non-zero element $m$ of $H_{d}^{t}(M)$ such that $\mathfrak{p}=\operatorname{Ann}(m)$ so that $\mathfrak{p} \in V(\Phi)$ by the pervious paragraph, because $H_{d}^{t}(M)$ is a $d$-torsion $R$-module. Thus $m \in$ $0:_{H_{d}^{t}(M)} \mathfrak{p} \cong \operatorname{Hom}_{R}\left(R / \mathfrak{p}, H_{d}^{t}(M)\right)$ and therefore $\bigcup_{\mathfrak{p} \in V(\Phi)} \operatorname{Ass}\left(\operatorname{Hom}_{R}\left(R / \mathfrak{p}, H_{d}^{t}(M)\right)\right)$ $\supseteq \operatorname{Ass}\left(H_{d}^{t}(M)\right)$. The result follows now by the proof of Lemma 2.1, since

$$
\operatorname{Hom}_{R}\left(R / \mathfrak{p}, H_{d}^{t}(M)\right) \cong \operatorname{Ext}_{R}^{t}(R / \mathfrak{p}, M) \cong \operatorname{Hom}_{R}\left(R / \mathfrak{p}, H_{\mathfrak{p}}^{t}(M)\right) .
$$

Theorem 2.4. Let $M$ be an $R$-module and $t$ be an integer such that $H_{d}^{i}(M)=0$, for all $i<t$. Then $\operatorname{Ass}\left(H_{d}^{t}(M)\right) \subseteq\{\mathfrak{p}: \operatorname{dim} R / \mathfrak{p}=d\}$ if and only if $H_{c}^{t}(M)=0$ for all integers $c<d$.

Proof. Assume that $\operatorname{Ass}\left(H_{d}^{t}(M)\right) \nsubseteq\{\mathfrak{p}: \operatorname{dim} R / \mathfrak{p}=d\}$. Thus there exists a prime ideal $\mathfrak{p}$ in $\operatorname{Ass}\left(H_{d}^{t}(M)\right)$ such that $\operatorname{dim} R / \mathfrak{p}=c<d$. Now, the exact sequence $0 \longrightarrow \Gamma_{c}(R / \mathfrak{p}) \longrightarrow \Gamma_{c}\left(H_{d}^{t}(M)\right)$ and $\Gamma_{c}(R / \mathfrak{p})=R / \mathfrak{p}, \Gamma_{c}\left(H_{d}^{t}(M)\right) \cong H_{c}^{t}(M)$ show that $\mathfrak{p} \in \operatorname{Ass}\left(H_{c}^{t}(M)\right)$. Hence, $H_{c}^{t}(M) \neq 0$. The converse is true by Lemma 2.1 (ii).

\section{The non-vanishing theorems}

In this section, we shall provide some results concerning the vanishing and nonvanishing of $d$-local cohomology modules. Throughout $R$ is a local ring with maximal ideal $\mathfrak{m}$ and $d$ is a non negative integer.

We are now in a position to prove that the non-vanishing theorems in the $d$-local cohomology modules.

Theorem 3.1. Let $(R, \mathfrak{m})$ be a local ring and let $M$ be a non-zero finitely generated $R$-module of dimension $n$. Then there is at least one $j$ with $0 \leq j \leq d$ for which $H_{d}^{n-j}(M) \neq 0$. 
Proof. Since $\Gamma_{\mathfrak{m}}\left(\Gamma_{d}(M)\right) \cong \Gamma_{\mathfrak{m}}(M)$, so there is the Grothendieck spectral sequence

$$
E_{2}^{i, j}:=H_{\mathfrak{m}}^{i}\left(H_{d}^{j}(M)\right) \Rightarrow H_{\mathfrak{m}}^{i+j}(M)=E^{i+j} .
$$

We have $\operatorname{Supp}\left(H_{d}^{j}(M)\right) \subseteq \Phi$ and so $\operatorname{dim} H_{d}^{j}(M) \leq d$. Thus by [6, Theorem 6.1.2], $E_{2}^{i, j}=0$ for all $i>d$. There is a filtration $0 \subseteq F^{n} E^{n} \subseteq \cdots \subseteq F^{1} E^{n} \subseteq E^{n}$ with $E_{\infty}^{i, n-i} \cong F^{i} E^{n} / F^{i+1} E^{n}$. Then $F^{d+1} E^{n}=\cdots=F^{n} E^{n}=0$. If $E_{\infty}^{j, n-j}=0$, for all $j$ with $0 \leq j \leq d$, then $F^{d} E^{n}=\cdots=F^{0} E^{n}=E^{n}=0$ contrary to [6, Theorem 7.3.2]. So suppose that $E_{\infty}^{j, n-j} \neq 0$ for some $j$ with $0 \leq j \leq d$. Thus $E_{2}^{j, n-j} \neq 0$ and then $H_{d}^{n-j}(M) \neq 0$. The proof is therefore complete.

Theorem 3.2. Let $R$ be a complete ring with respect to the $\mathfrak{m}$-adic topology. Then the following statements are true:

(i) If $M$ is a finitely generated $R$-module of dimension $n$ and $0<d \leq n$, then $H_{d}^{n}(M)=0$.

(ii) If $\operatorname{dim} R=n$ and $0<d<n$, then either $H_{d}^{n-1}(R)=0$ or $H_{d}^{n-1}(R)$ is not finitely generated. In particular, $H_{1}^{n-1}(R)$ is not finitely generated.

Proof. (i) Let $\mathfrak{p} \in \operatorname{Ass}(M)$ and $\operatorname{dim} R / \mathfrak{p}=n$. In this case $\Phi$ contains an ideal $I$ of $R$ with $\operatorname{dim} R / I=1$ and $\mathfrak{p} \subseteq I$. So that $\operatorname{dim} R /(I+\mathfrak{p})=\operatorname{dim} R / I=1$ thus in view of [13, Theorem 2.4], the result follows.

(ii) Let $H_{d}^{n-1}(R) \neq 0$. Then $H_{d}^{n-1}(R) \cong H_{d}^{n-1}\left(R / \Gamma_{d}(R)\right)$. So that in view of [6, Lemma 2.1.1] there exists a non-zero divisor $x \in \mathfrak{m}$. The exact sequence $0 \rightarrow$ $R \stackrel{x}{\rightarrow} R \rightarrow R / x R \rightarrow 0$ induces an exact sequence $\cdots \rightarrow H_{d}^{n-1}(R) \stackrel{x}{\rightarrow} H_{d}^{n-1}(R) \rightarrow$ $H_{d}^{n-1}(R / x R)$. By assumption $R / x R$ is a local complete ring of dimension $n-1$, so that $H_{d}^{n-1}(R / x R)=0$ by (i). Thus $H_{d}^{n-1}(R)=x H_{d}^{n-1}(R)$ which implies $H_{d}^{n-1}(R)$ is not finitely generated by Nakayma's Lemma.

In view of Theorem 3.1, there exists $j$ with $0 \leq j \leq 1$ such that $H_{1}^{n-j}(R) \neq 0$ on the other hand $H_{1}^{n}(R)=0$ by (i). Hence, $H_{1}^{n-1}(R) \neq 0$ and so is not finitely generated.

Corollary 3.3. If $R$ is a complete ring with respect to the $\mathfrak{m}$-adic topology and $M$ is a non-zero finitely generated $R$-module of dimension $n \geq 1$, then there is at least one $j$ with $1 \leq j \leq d$ for which $H_{d}^{n-j}(M) \neq 0$.

Proof. It is obvious by Theorems 3.1 and 3.2.

Theorem 3.4. Let $R$ be Gorenstein of dimension $n$ and let $0 \leq d \leq n$. Then the following statements are true:

(i) $H_{d}^{i}(R) \neq 0$ if and only if $i=n-d$. 
(ii) $\operatorname{Ass}\left(H_{d}^{n-d}(R)\right)=\{\mathfrak{p} \in \operatorname{Spec}(R): \operatorname{dim} R / \mathfrak{p}=d\}$.

(iii) $H_{d}^{n-d}(R)$ is not an Artinian module, for $d>0$.

(iv) $H_{d}^{n-d}(R)$ has injective dimension $d$.

(v) $H_{d}^{n-d}(R)$ is a flat module.

Proof. (i) Let $0 \rightarrow R \rightarrow E^{0} \rightarrow E^{1} \rightarrow \cdots \rightarrow E^{n-1} \rightarrow E^{n} \rightarrow 0$ be a minimal injective resolution of $R$. Then by [12, Theorems 18.1 and 18.8] we have $E^{i}=$ $\bigoplus_{\operatorname{dim} R / \mathfrak{p}=n-i} E(R / \mathfrak{p})$. If $i \geq n-d$, then $\operatorname{dim} R / \mathfrak{p}=n-i \leq n-(n-d)=d$, and so $\mathfrak{p} \in \Phi$. Thus $\Gamma_{d}(E(R / \mathfrak{p}))=E(R / \mathfrak{p})$ and therefore $\Gamma_{d}\left(E^{i}\right)=E^{i}$. If $i<n-d$, then $\operatorname{dim} R / \mathfrak{p}=n-i>d$ and so $\mathfrak{p} \notin \Phi$. Thus $\Gamma_{d}(E(R / \mathfrak{p}))=0$ and then $\Gamma_{d}\left(E^{i}\right)=0$. It follows that $H_{d}^{i}(R)=0$, whenever $i \neq n-d$. On the other hand, by Theorem 3.1, there is one $j$ with $0 \leq j \leq d$ for which $H_{d}^{n-j}(R) \neq 0$. Hence, $H_{d}^{n-d}(R) \neq 0$.

(ii) This is immediate from (i) that

$$
\operatorname{Ass}\left(H_{d}^{n-d}(R)\right) \subseteq \operatorname{Ass}\left(\bigoplus_{\operatorname{dim} R / \mathfrak{p}=d} E(R / \mathfrak{p})\right)=\{\mathfrak{p} \in \operatorname{Spec}(R): \operatorname{dim} R / \mathfrak{p}=d\} .
$$

Let $\mathfrak{p} \in \operatorname{Spec}(R)$ and $\operatorname{dim} R / \mathfrak{p}=d$. By [2, Lemma], $\left(H_{d}^{n-d}(R)\right)_{\mathfrak{p}} \cong H_{d-\operatorname{dim} R / \mathfrak{p}}^{n-d}\left(R_{\mathfrak{p}}\right)=$ $H_{0}^{n-d}\left(R_{\mathfrak{p}}\right) \cong H_{\mathfrak{p} R_{\mathfrak{p}}}^{n-d}\left(R_{\mathfrak{p}}\right)$ since every Gorenstein local ring is catenary and biequidimensional, see [12, Theorem 17.3]. Moreover, $H_{\mathfrak{p} R_{\mathfrak{p}}}^{n-d}\left(R_{\mathfrak{p}}\right) \neq 0$, by [6, Theorem 7.3.2]. Thus $\mathfrak{p}$ is a minimal element of $\operatorname{Supp}\left(H_{d}^{n-d}(R)\right)$ and then $\mathfrak{p} \in \operatorname{Ass}\left(H_{d}^{n-d}(R)\right)$.

(iii) By (ii), $\operatorname{Ass}\left(H_{d}^{n-d}(R)\right) \nsubseteq \mathbb{M a x}(R)$ so $H_{d}^{n-d}(R)$ is not Artinian.

(iv) It is obvious by the proof of (i).

(v) See [17, Theorem 2.1].

Example 3.5. Let $K$ be a field and let $R=K\left[X_{1}, \cdots, X_{n}\right]$ be the ring of polynomials over $K$ in the indeterminates $X_{1}, \cdots, X_{n}$. Then for $\mathfrak{m}=\left(X_{1}, \cdots, X_{n}\right), R_{\mathfrak{m}}$ is a local Goenstein ring of dimension n. So by Theorem 3.4 we have

$$
\operatorname{Ass}\left(H_{1}^{n-1}\left(R_{\mathfrak{m}}\right)\right)=\left\{\mathfrak{p} R_{\mathfrak{m}} \in \operatorname{Spec}\left(R_{\mathfrak{m}}\right): \operatorname{dim}(R / \mathfrak{p})_{\mathfrak{m}}=1\right\} .
$$

Now, $\operatorname{Ass}\left(H_{1}^{n-1}\left(R_{\mathfrak{m}}\right)\right)$ has infinite members by [15, Exercise 15.3].

Theorem 3.6. Let $R$ be Gorenstein of dimension $n, I$ an ideal of $R$ and let $0 \leq$ $d \leq n$. Then the following statements are true:

(i) If $I \notin \Phi$, then for all $i \geq 0, H_{I}^{i}\left(H_{d}^{n-d}(R)\right) \cong H_{\Psi}^{n-d+i}(R)$, where $\Psi=$ $\left\{I^{n}+J: n \geq 1, J \in \Phi\right\}$.

(ii) For all $I \in \Phi$ and all $i \geq 0, H_{I}^{i}\left(H_{d}^{n-d}(R)\right) \cong H_{I}^{n-d+i}(R)$.

Proof. (i) Let $I \notin \Phi$ and $\Psi=\left\{I^{n}+J: n \geq 1, J \in \Phi\right\}$. Then $\Gamma_{I}\left(\Gamma_{d}(R)\right)=\Gamma_{\Psi}(R)$. Thus there is a Grothendieck spectral sequence

$$
E_{2}^{i, j}:=H_{I}^{i}\left(H_{d}^{j}(R)\right) \Longrightarrow H_{\Psi}^{i+j}(R)=E^{i+j} .
$$


Since $E_{\infty}^{i, n-d-i}$ is a subquotient of $E_{2}^{i, n-d-i}$ so by Theorem 3.4(i) we have $E_{\infty}^{i, n-d-i}=$ 0 , for all $i \geq 1$. Thus the filtration

$$
0=F^{n-d} E^{n-d} \subseteq F^{n-d-1} E^{n-d} \subseteq \cdots \subseteq F^{1} E^{n-d} \subseteq F^{0} E^{n-d}=E^{n-d}
$$

implies that $F^{n-d} E^{n-d}=\cdots=F^{1} E^{n-d}=0$ and $E_{\infty}^{0, n-d} \cong E^{n-d}$. By the sequence $0 \longrightarrow E_{2}^{0, n-d} \longrightarrow E_{2}^{2, n-d-1}$ and $E_{2}^{2, n-d-1}=0$ we have $E_{\infty}^{0, n-d} \cong E_{2}^{0, n-d}$. Therefore, $\Gamma_{I}\left(H_{d}^{n-d}(R)\right) \cong H_{\Psi}^{n-d}(R)$.

Let $0 \rightarrow R \rightarrow E^{0} \rightarrow E^{1} \rightarrow \cdots \rightarrow E^{n-1} \rightarrow E^{n} \rightarrow 0$ be a minimal injective resolution of $R$. Then $0 \rightarrow H_{d}^{n-d}(R) \rightarrow E^{n-d} \rightarrow \cdots \rightarrow E^{n-1} \rightarrow E^{n} \rightarrow 0$ is an injective resolution of $H_{d}^{n-d}(R)$. For all $i \geq n-d$ we have

$$
\Gamma_{I}\left(E^{i}\right) \cong \oplus_{\operatorname{dim} R / \mathfrak{p}=n-i} \Gamma_{I}(E(R / \mathfrak{p})) \cong \oplus_{\operatorname{dim} R / \mathfrak{p}=n-i} \Gamma_{\Psi}(E(R / \mathfrak{p})) \cong \Gamma_{\Psi}\left(E^{i}\right)
$$

Hence, $H_{I}^{i}\left(H_{d}^{n-d}(R)\right) \cong H_{\Psi}^{n-d+i}(R)$, for all $i>0$.

(ii) The proof is similar to that of (i).

Corollary 3.7. Let $R$ be Gorenstein of dimension $n$ and let $0 \leq d \leq n$. Then $\operatorname{dim} H_{d}^{n-d}(R)=\operatorname{depth} H_{d}^{n-d}(R)=d$ and $H_{d}^{n-d}(R)$ is not a finitely generated $R$ module.

Proof. It follows by the proof of Theorems 3.2(ii), 3.4, 3.6 and [7, Exercise 9.1.12(c)].

Lemma 3.8. Let $\left\{\mathfrak{p}_{\lambda}: \mathfrak{p}_{\lambda} \notin \Phi\right\}_{\lambda \in \Lambda}$ be a family of prime ideals of $R$. Then for any $d$-torsion $R$-module $M, \operatorname{Hom}_{R}\left(M, \oplus_{\lambda \in \Lambda} E\left(R / \mathfrak{p}_{\lambda}\right)\right)=0$.

Proof. Suppose that $\operatorname{Hom}_{R}(M, E(R / \mathfrak{p})) \neq 0$, for some $\mathfrak{p} \notin \Phi$. Thus there is a nonzero element $f \in \operatorname{Hom}_{R}(M, E(R / \mathfrak{p}))$ so $f(x) \neq 0$ for some $x \in M$. By assumption there is an $I \in \Phi$ such that $I x=0$ and so $I f(x)=0$. Hence, $I \subseteq \mathfrak{p}$. Otherwise for each $a \in I \backslash \mathfrak{p}$ we have the automorphism $E(R / \mathfrak{p}) \stackrel{a}{\rightarrow} E(R / \mathfrak{p})$, contrary to $\operatorname{If}(x)=0$. Therefore, $\operatorname{Hom}_{R}(M, E(R / \mathfrak{p}))=0$ and so $\operatorname{Hom}_{R}\left(M, \oplus_{\lambda \in \Lambda} E\left(R / \mathfrak{p}_{\lambda}\right)\right)=0$.

Theorem 3.9. Let $R$ be Gorenstein of dimension $n$ and let

$$
0 \rightarrow R \rightarrow E^{0} \rightarrow \cdots \rightarrow E^{i} \stackrel{d^{i}}{\rightarrow} E^{i+1} \cdots \rightarrow E^{n-1} \rightarrow E^{n} \rightarrow 0
$$

be an injective resolution of $R$. Then the following statements are true:

(i) $\operatorname{Ext}_{R}^{j}\left(H_{d}^{i}(M), R\right)=0$, for all $R$-module $M, 0 \leq j<n-d$ and $i \geq 0$.

(ii) $\operatorname{Ext}_{R}^{j}\left(E^{i}, R\right)=0$ for all $i \geq 1$ and $j<i$.

Proof. (i) It follows by Lemma 3.8. 
(ii) For $i \geq 1$ we have $H_{n-i}^{i}(R) \cong \operatorname{ker} d^{i}$. Thus

$$
0 \rightarrow H_{n-i}^{i}(R) \rightarrow E^{i} \rightarrow H_{n-i-1}^{i+1}(R) \rightarrow 0
$$

is an exact sequence that induces the long exact sequence

$$
\cdots \rightarrow \operatorname{Ext}_{R}^{j}\left(H_{n-i-1}^{i+1}(R), R\right) \rightarrow \operatorname{Ext}_{R}^{j}\left(E^{i}, R\right) \rightarrow \operatorname{Ext}_{R}^{j}\left(H_{n-i}^{i}(R), R\right) \rightarrow \cdots .
$$

By (i), $\operatorname{Ext}_{R}^{j}\left(H_{n-i-1}^{i+1}(R), R\right)=0=\operatorname{Ext}_{R}^{j}\left(H_{n-i}^{i}(R), R\right)$, for all $j<i$. Hence $\operatorname{Ext}_{R}^{j}\left(E^{i}, R\right)=0$ for all $j<i$

Acknowledgement. The authors would like to thank the referee for the valuable suggestions and comments.

\section{References}

[1] J. Azami, R. Naghipour and B. Vakili, Finiteness properties of local cohomology modules for a-minimax modules, Proc. Amer. Math. Soc., 137(2) (2009), 439448.

[2] C. Banica and M. Stoia, Singular sets of a module and local cohomology, Boll. Un. Mat. Ital. B, 16 (1976), 923-934.

[3] M. H. Bijan-Zadeh, Torsion theories and local cohomology over commutative Noetherian rings, J. London Math. Soc., 19(3) (1979), 402-410.

[4] K. B. Lorestani, P. Sahandi and S. Yassemi, Artinian local cohomology modules, Canad. Math. Bull., 50(4) (2007), 598-602.

[5] M. P. Brodmann and A. L. Faghani, A finiteness result for associated primes of local cohomology modules, Proc. Amer. Math. Soc., 128(10) (2000), 2851-2853.

[6] M. P. Brodmann and R. Y. Sharp, Local Cohomology: an Algebraic Introduction with Geometric Applications, Cambridge Studies in Advanced Mathematics, 60, Cambridge University Press, Cambridge, 1998.

[7] W. Bruns and J. Herzog, Cohen-Macaulay Rings, Cambridge Studies in Advanced Mathematics, 39, Cambridge University Press, Cambridge, 1993.

[8] M. T. Dibaei and S. Yassemi, Associated primes and cofiniteness of local cohomology modules, Manuscripta Math., 117(2) (2005), 199-205.

[9] K. Divaani-Aazar and A. Mafi, Associated primes of local cohomology modules, Proc. Amer. Math. Soc., 133(3) (2005), 655-660.

[10] C. Huneke, Problems on local cohomology. Free Resolution in Commutative Algebra and Algebraic Geometry. (Sundance, UT, 1990), Res. Notes Math., 2, Jones and Bartlett, Boston, MA, (1992), 93-108.

[11] M. Katzman, An example of an infinite set of associated primes of local cohomology module, J. Algebra, 252(1) (2002), 161-166. 
[12] H. Matsumura, Commutative Ring Theory, Cambridge Studies in Advanced Mathematics, 8, Cambridge University Press, Cambridge, 1986.

[13] R. Naghipour, Integral closures, local cohomology and ideal topologies, Rocky Mountain J. Math., 37(3) (2007), 905-916.

[14] J. J. Rotman, Introduction to Homological Algebra, Pure and Applied Mathematics, 85, Academic Press, Inc. [Harcourt Brace Jovanovich, Publishers], New York-London, 1979.

[15] R. Y. Sharp, Steps in Commutative Algebra, Second edition, London Mathematical Society Student Texts, 51, Cambridge University Press, Cambridge, 2000.

[16] A. K. Singh, p-Torsion elements in local cohomology modules, Math. Res. Lett., $7(2-3)$ (2000), 165-176.

[17] J. Z. Xu, Minimal injective and flat resolutions of modules over Gorenstein rings, J. Algebra, 175(2) (1995), 451-477.

[18] N. Zamani, M. H. Bijan-Zadeh and M. S. Sayedsadeghi, $d$-Transform functor and some finiteness and isomorphism results, Vietnam. J. Math., 42(2) (2014), 179-186.

[19] N. Zamani, M. H. Bijan-Zadeh and M. S. Sayedsadeghi, Cohomology with support of dimension $\leq d$, J. Algebra Appl., 15(3) (2016), 1650042 (10 pp).

Z. Rahimi-Molaei, Sh. Payrovi (Corresponding Author) and S. Babaei

Department of Mathematics

Imam Khomeini International University

Postal Code: 34149-1-6818

Qazvin, Iran

e-mails: z.rahimi@edu.ikiu.ac.ir (Z. Rahimi-Molaei)

shpayrovi@sci.ikiu.ac.ir (Sh. Payrovi)

sakinehbabaei@gmail.com (S. Babaei) 